\title{
Brain Metastasis of Breast Cancer: Crossing the Blood-brain Barrier
}

\author{
Jieliang Li, PhD* \\ Department of Pathology and Laboratory Medicine, \\ Temple University Lewis Katz School of Medicine, Philadelphia, PA, USA
}

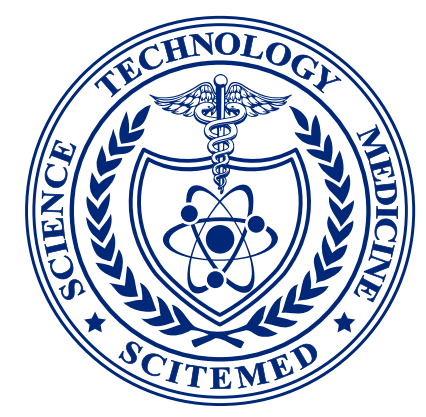

\begin{abstract}
Breast cancers are the most common cancers in women and are associated with a relatively high rate of brain metastases, causing a severe increase in mortality. Brain metastases most often appear at the areas of white and gray junctions, which contain cerebral blood vessels. Recent studies have revealed that the metastatic breast cancers characteristically express and secrete a number of factors, including exosome-associated or circulating microRNAs (miRNAs). These factors can destroy the tight junctions formed by brain microvascular endothelial cells and the integrity of the blood-brain barrier (BBB), enabling the breast cancer cells to invade into the brain tissue. In addition, the metastatic breast cancer cells in brain express high levels of anti-plasminogen activator (PA) serpins as a shield to escape from the reaction of brain stroma, conferring the adherence of infiltrating breast cancer cells to the surface of capillaries and the growth on the vasculature. This mini review centers on the recent findings of miRNAs in breast cancers brain metastasis and the active crosstalk with the BBB.
\end{abstract}

\section{Introduction}

Cells originating from peripheral primary tumors can circulate in the blood flow to the brain and form brain metastases, which are also known as secondary brain cancers. Metastatic brain tumors occur in about $5-25 \%$ of all cancers that spread through the body and are about 10 times more common than the cancers that start in the brain (primary brain cancers) [1]. Primary cancers that originate from the lung, breast, colon, and kidney, along with malignant melanoma, are the most likely to metastasize to the brain [2,3]. Lung cancers and breast cancers account for nearly two-third of the total cases of brain metastases.

Breast cancers are the most common cancers in women in the United States and a leading cause of cancer death among women [4]. While systemic therapy reduces the risk of recurrence, many patients still develop metastatic disease. In some studies, about $20 \%-40 \%$ of the patients with breast cancers treated with chemotherapeutic and/or hormonal agents eventually develop recurrences in distant organs with a low 5 -year survival rate of only $\sim 20 \%$ [5-8]. Breast cancers are associated with a relatively high rate of brain metastases carrying total incidence about $30 \%$ [9]. Despite the improvement of primary breast cancer treatments in the past few decades and as a consequence of prolonged life span, the number of brain metastases is rising in patients with breast cancers [10]. In addition, due to the low permeability of most chemotherapies to the brain, metastasis of breast cancers to the brain is invariably associated with poor prognosis and causes a severe increase in mortality.

\section{Diagnosis and Treatment of Brain Metastatic Breast Cancers}

As metastatic tumors originate from breast, the diagnosis of brain metastatic breast cancer is different from primary brain tumors, infectious processes, and other central nervous system (CNS) lesions. The current diagnoses for brain metastatic breast cancer include contrast-enhanced magnetic resonance imaging (MRI) and biopsy. MRI is the preferred imaging study for the diagnosis of brain metastases [11]. With respect to detection, localization, and quantification, contrast enhanced MRI has been widely demonstrated to be more sensitive than either non-enhanced MRI or computed tomography (CT) scanning [11,12]. Newer modalities in radiographic imaging, such as magnetization transfer (MT) [13], triple dose gadolinium imaging [14], echo planar imaging [15], MR spectroscopy [16], positron emission tomography (PET) [17], and single-photon emission computerized tomography (SPECT) [18] have further improved brain lesion detection with MRI. Nevertheless, biopsy remains the gold standard to establish the diagnosis of brain metastases. Biopsy is par- ticularly important in patients with a single lesion to exclude the primary brain tumors or lesions and to identify the breast origins of brain metastases. The risk of CNS relapse among patients with breast cancer varies significantly by tumor stage with less than $3 \%$ among women presenting with early-stage breast cancers, while $10-16 \%$ in patients with metastatic breast cancers [19].

Although breast cancers are top sources of brain metastasis, most current management algorithms for brain metastatic breast cancers are based upon guidelines for secondary brain metastases in general, rather than being specific for breast cancers [20]. The stages of breast cancers, the prior chemotherapy, and CNS radiation exposure have all confounded the therapy regimens and results [21]. Novel chemotherapy agents (e.g. Canazitaxel, Iromptecan) and therapeutic strategies targeting the human epidermal growth factor rector 2 (HER2) (e.g. Lapatinib, Afatinib), mTOR (e.g. Everolimus), PI3K (e.g. BKM120), PARP (e.g. Sorafenib), and VEGF (e.g. GRN1005) are under evaluation for the treatment of brain metastatic breast cancers. Systemic benefit is emphasized in these specifically molecular subtyping targeted therapies [22]. For example, HER2-positive breast cancers demonstrate a tropism for CNS spread [23]. Clinical trials using inhibitors targeting HER2 have witnessed the increase in the time for brain metastases to develop and decrease the frequency of CNS involvement at progression [24,25]. However, Herceptin (chemical name: trastuzumab) has limited ability to penetrate the blood-brain barrier (BBB) and is less effective in preventing the development of cerebral disease [26]. Therefore, new therapies focusing on the prevention of brain metastases and/or on increasing the permeability of the BBB to allow systemic therapies to reach the brain should be sought.

\section{Blood-brain Barrier and Brain Metastatic Breast Cancers}

Brain metastasis is a multistep process that involves detachment of tumor cells from the primary lesion, followed by survival in the blood vessels or lymphatic system, invasion into the CNS, and sustained proliferation towards the formation of brain tumor. Metastatic breast cancer cells can break away from the primary tumor site and travel through blood and lymphatic vessels. According to the "seed and soil" hypothesis for organ-preference patterns of tumor metastasis (metastatic tumor cells as "seed"; their organ microenvironment as "soil") proposed by Paget [27], the migratory cancer cells leave the primary site via intravasation, circulate throughout the body and eventually engraft in distant organs, which allow proper adherence and colonization. The "soil" for malignant cells in the CNS has been suggested to be vascular rather than neuronal 
[28-30]. Therefore, the consecutive steps in brain metastasis require sophisticated communication between the metastatic cancerous cells and brain, particularly at the capillary level.

The BBB is a selective barrier that lines cerebral micro-vessels and actively controls the passage of substances and circulating cells from the blood into the cerebrospinal fluid. The BBB deliberately maintains the CNS homeostasis. A key aspect of BBB is the formation of the complex tight junctional capillaries by brain microvascular endothelial cells (BMVEC). Given the unique functions of the brain, the structure of BBB is distinct from endothelial barriers in other organs. For example, the capillaries in the bone marrow and the liver are fenestrated [31,32], whereas the tight junctional capillaries by BMVEC are backed by the "end feet" of astrocytes in association with neurons, forming the functional neurovascular units [33]. The compromised function of BBB also represents a key early event in the pathogenesis of several neurodegenerative disorders with an inflammatory component, such as Alzheimer's disease and Parkinson's disease [34].

Recent studies showed that the brain metastasis of breast cancers involves mediators of extravasation through non-fenestrated capillaries. Brain metastasis is complemented by specific enhancers of BBB crossing and brain colonization. A number of mediators have been identified to assist breast cancer cells crossing the BBB, including cyclooxygenase (COX-2), epidermal growth factor receptor (EGFR) ligand HBEGF, and the $a 2,6$-sialyltransferase ST6GALNAC5 [35]. COX-2 and EGFR are also known to be involved in the infiltration of breast cancer cells into the lung [36,37], suggesting a sharing of these mediators in cerebral and pulmonary metastases. In contrast, ST6GALNAC5 was found to specifically mediate breast cancer brain metastasis [35]. In addition, the leukocyte adhesion molecule CD44 [38] and the vascular endothelial growth factor (VEGF) [39] also contribute to the adhesion of metastatic breast cancer cells to brain vascular endothelium and trans-endothelial migration across the BBB.

\section{Dysregulated Mirna Secretion of Brain Metastatic Breast Cancers}

MicroRNAs (miRNAs) are small non-coding RNAs that are involved in post-transcriptional gene regulation. Recent work has shown that miRNAs of cancer cells modulate the tumor microenvironment, such as tumor angiogenesis, tumor immune invasion, and tumor-stromal interactions [40]. In breast cancer cells, miR-9 can target and downregulate E-cadherin, which in turn upregulates VEGF expression, leading to the increase of cell motility and invasiveness [41]. Furthermore, miR-210 secreted from breast cancer cells was found to serve as an angiogenic miRNA in recipient endothelial cells [42]. Plasma miR-21 was also identified to be elevated in patients with breast cancers. MiR-21 has been implicated to promote the tumor cell proliferation and invasion by suppressing smad7, which enhances EGF and TGF- $\beta$ pathways [43]. In contrast, miR126 [44] and miR-29b [45] were shown to non-cell-autonomously restrict breast cancer metastasis via regulation of multiple targets involved in endothelial recruitment or extracellular matrix remodeling.

Regarding the brain metastasis of breast cancers, Zhou et al. [46], for the first time, identified that the transwell migration of primary BMVEC was significantly stimulated by exosomes from MDA-231 metastatic breast cancer cell line, but not the noncancerous mammary epithelial cell line MCF-10A. Using the deep sequencing technique, they identified a number of miRNAs differentially secreted in exosomes between the two lines, particularly miR-105. The exosomal enriched miR-105 from MDA231 cells was predicted to target multiple sites on the mRNAs of tight junction proteins. A panel of metastatic breast cancer cell lines isolated from pleural effusion was further characterized to secrete high levels of exosomal miR-105. Indeed, miR-105 was identified to be involved in a cancer-germline transcript (CT-GABRA3) displaying DNA hypomethylation-dependent activation in various tumors [47]. The serum level of neutral sphingomyelinase 2 (nSMase 2), which regulates the exosomal miRNA secretion, was found to be enhanced in a breast cancer model previously [42]. The serum miR-105 levels, correlated with the progression of distant metastases of clinical breast cancers, suggest a predicator role of miR-105 in the diagnosis of brain metastasis. Therefore, the metastatic breast cancer cells have the potential to secrete miR-105 in exo- somes as paracrine or endocrine regulatory mechanism to break away from the primary tumor site and travel through blood and lymphatic vessels of distant organs.

Besides miR-105, miR-10b was also reported in the invasion and metastasis of breast cancers [48]. Singh et al. revealed that miR-10b was very highly expressed in exosomes secreted by metastatic breast cancer cells [49]. Upon uptake by recipient cells, miR-10b can suppress the protein level of its target genes, such as HOXD10 and KLF4. These two factors are involved in the regulation of cell proliferation and differentiation, promoting the invasive ability of non-malignant breast cancer cells. Most recently, metastatic breast cancer cells were identified to secrete high circulating miR-122 levels [50]. MiR-122 can suppress the glucose uptake by non-tumor cells in the premetastatic niche to promote metastasis [50]. Inhibition of miR-122 could restore glucose uptake in distant organs, including brain and lungs, and decrease the incidence of metastasis.

\section{Evasion of Brain Defense by Metastatic Breast Cancers}

The recently identified extracellular (circulating or exosomal) miRNAs were shown to mediate the ability of metastatic breast cancer cells to target the distant brain endothelium and vasculature. However, brain metastasis tends to be a late complication of cancer in the clinic $[51,52]$. Most cancer cells that leave a solid tumor perish, and much of this attrition happens as circulating cancer cells infiltrate distant organs [53]. Real-time imaging [54] and MRI [55] techniques revealed that most cancer cells that pass the BBB die. This means that during or following the migration and transverse, the breast cancer cells will face local defense from the brain. Valiente et al. [56] recently showed a preventive mechanism of the brain against cancer cell brain metastases. The reactive brain stroma expresses plasmin as a defense against the metastatic invasion of breast cancers in two ways: first, plasmin can convert the membrane-bound astrocytic FasL into a paracrine death signal for invading tumor cells, mediating the killing of the metastatic cells. Second, plasmin prevents vascular cooption by inactivating the axon pathfinding molecule L1 cell adhesion molecule (L1CAM) expressed in metastatic cells. This L1CAM inhibition prevents the adherence of breast cancer cells to the surface of endothelial capillaries and growth as a sheath around the vessels. Interestingly, breast cancer cells of brain metastatic phenotype express upregulated levels of neuroserpin (NS) and serpin B2 as compared to their parental cells. NS and serpin B2 are associated with plasminogen activator (PA)-inhibitory activity, which enables the metastatic cells to evade the suppression by plasmin. NS was previously believed to be restricted to neurons where it protects against PA cytotoxicity from reactive astrocytes [57]. Indeed, cancer cells infiltrate the brain and are immediately exposed to astrocytes that abound in the perivascular space and produce either the death signals to invading cells or factors to prevent the colonization of metastatic cells from the brain. Therefore, the increased expression of serpins by disseminated breast cancer cells provides an adaptation for successful brain metastasis.

\section{Conclusion}

The high incidence of brain metastasis and the comparison of metastatic and non-metastatic phenotypes indicate an active crosstalk of brain metastatic breast cancers with the BBB. Certain miRNAs and serpins are regulatory molecules in defining the metastatic potential of breast cancers. Targeting these factors that favor the metastatic microenvironment may provide future therapeutic interventions for the brain metastasis of breast cancers.

\author{
Article Information \\ *Correspondence: Jieliang Li, PhD \\ Department of Pathology and Laboratory Medicine, Temple University \\ Lewis Katz School of Medicine, Philadelphia, PA, USA. \\ Email: jieliang.li@temple.edu
}

Received:June 16, 2017; Accepted: Oct. 03, 2017; Published: Dec. 06, 2017 
Copyright (c) 2017 The Author. This is an open-access article distributed under the terms of the Creative Commons Attribution 4.0 International License (CC-BY).

Funding: This work was supported by the National Institutes of Health [DA040329 and $\mathrm{MH} 109385]$.

\section{Conflict of Interest Disclosures: None}

\section{Keywords}

Brain metastases; breast cancer; exosome; miRNAs; tight junction; bloodbrain barrier.

\section{References}

1. Maher EA, Mietz J, Arteaga CL, DePinho RA, Mohla S. Brain metastasis: opportunities in basic and translational research. Cancer Research 2009;69:6015-6020.

2. Fidler IJ, Balasubramanian K, Lin Q, Kim SW, Kim SJ. The brain microenvironment and cancer metastasis. Molecules and Cells 2010;30:9398.

3. Eichler AF, Chung E, Kodack DP, Loeffler JS, Fukumura D, Jain RK. The biology of brain metastases-translation to new therapies. Nature Reviews Clinical Oncology 2011;8:344-356.

4. American Cancer Society. Cancer facts and statistics. Available at: https://www.cancer.org/

5. Moreno-Aspitia A, Perez EA. Treatment options for breast cancer resistant to anthracycline and taxane. Mayo Clinic Proceedings 2009;84:533-545.

6. Nicolini A, Giardino R, Carpi A, et al. Metastatic breast cancer: an updating. Biomedicine \& pharmacotherapy 2006;60:548-556.

7. Yardley DA. Visceral disease in patients with metastatic breast cancer: efficacy and safety of treatment with ixabepilone and other chemotherapeutic agents. Clinical Breast Cancer 2010;10:64-73.

8. Zhang XH, Giuliano M, Trivedi MV, Schiff R, Osborne CK. Metastasis dormancy in estrogen receptor-positive breast cancer. Clinical Cancer Research: An Official Journal of the American Association for Cancer Research 2013;19:6389-6397.

9. Cheng X, Hung MC. Breast cancer brain metastases. Cancer Metastasis Reviews 2007;26:635-643.

10. Wikman H, Westphal L, Schmid F, et al. Loss of CADM1 expression is associated with poor prognosis and brain metastasis in breast cancer patients. Oncotarget 2014;5:3076-3087.

11. Fink KR, Fink JR. Imaging of brain metastases. Surgical Neurology International 2013;4:S209-219.

12. Zakaria R, Das K, Bhojak M, Radon M, Walker C, Jenkinson MD. The role of magnetic resonance imaging in the management of brain metastases: diagnosis to prognosis. Cancer Imaging: the Official Publication of the International Cancer Imaging Society 2014;14:8.

13. Haba D, Pasco Papon A, Tanguy JY, Burtin P, Aube C, Caron-Poitreau C. Use of half-dose gadolinium-enhanced MRI and magnetization transfer saturation in brain tumors. European Radiology 2001;11:117122.

14. Sze G, Johnson C, Kawamura Y, et al. Comparison of single- and triple-dose contrast material in the MR screening of brain metastases. AJNR American Journal of Neuroradiology 1998;19:821-828.

15. Young GS, Setayesh K. Spin-echo echo-planar perfusion MR imaging in the differential diagnosis of solitary enhancing brain lesions: distinguishing solitary metastases from primary glioma. AJNR American Journal of Neuroradiology 2009;30:575-577.

16. Bulakbasi N, Kocaoglu M, Ors F, Tayfun C, Ucoz T. Combination of single-voxel proton MR spectroscopy and apparent diffusion coefficient calculation in the evaluation of common brain tumors. AJNR American journal of Neuroradiology 2003;24:225-233.

17. Lu Y, FDG and ${ }^{82} \mathrm{Rb}$ PET/MRI features of brain metastasis of breast cancer. Clinical Nuclear Medicine 2015;40:494-495.
18. Palumbo B, Buresta T, Nuvoli S, et al. SPECT and PET serve as molecular imaging techniques and in vivo biomarkers for brain metastases. International Journal of Molecular Sciences 2014;15:9878-9893.

19. Barnholtz-Sloan JS, Sloan AE, Davis FG, Vigneau FD, Lai P, Sawaya RE. Incidence proportions of brain metastases in patients diagnosed (1973 to 2001) in the metropolitan Detroit cancer surveillance system. Journal of Clinical Oncology: Official Journal of the American Society of Clinical Oncology 2004;22:2865-2872.

20. Lim E, Lin NU. Updates on the management of breast cancer brain metastases. Oncology 2014;28:572-578.

21. Witzel I, Oliveira-Ferrer L, Pantel K, Muller V, Wikman H. Breast cancer brain metastases: biology and new clinical perspectives. Breast Cancer Research: BCR 2016;18:8.

22. Renfrow JJ, Lesser GJ. Molecular subtyping of brain metastases and implications for therapy. Current Treatment Options in Oncology 2013;14:514-527.

23. Venur VA, Leone JP. Targeted therapies for brain metastases from breast cancer. International Journal of Molecular Sciences 2016;17(9):1543.

24. Verma S, Miles D, Gianni L, et al. Trastuzumab emtansine for HER2-positive advanced breast cancer. The New England Journal of Medicine 2012;367:1783-1791

25. Baselga J, Cortes J, Kim SB, et al. Pertuzumab plus trastuzumab plus docetaxel for metastatic breast cancer. The New England Journal of Medicine 2012;366:109-119.

26. El-Habashy SE, Nazief AM, Adkins CE, et al. Novel treatment strategies for brain tumors and metastases. Pharmaceutical Patent Analyst 2014;3:279-296.

27. Langley RR, Fidler IJ. The seed and soil hypothesis revisited--the role of tumor-stroma interactions in metastasis to different organs. International Journal of Cancer Journal 2011;128:2527-35.

28. Ramakrishna R, Rostomily R. Seed, soil, and beyond: the basic biology of brain metastasis. Surgical Neurology International 2013;4:S256-S264.

29. Liu Y, Kosaka A, Ikeura M, et al. Premetastatic soil and prevention of breast cancer brain metastasis. Neuro-Oncology 2013;15:891-903.

30. Carbonell WS, Ansorge O, Sibson N, Muschel R. The vascular basement membrane as "soil" in brain metastasis. PLOS One 2009;4(6):e5857.

31. Paku S, Dome B, Toth R, Timar J. Organ-specificity of the extravasation process: an ultrastructural study. Clinical \& Experimental Metastasis 2000;18:481-492.

32. Inoue S, Osmond DG. Basement membrane of mouse bone marrow sinusoids shows distinctive structure and proteoglycan composition: a high resolution ultrastructural study. The Anatomical Record 2001;264(3):294-304.

33. Banerjee $S$, Bhat MA. Neuron-glial interactions in blood-brain barrier formation. Annual Review of Neuroscience 2007;30:235-258.

34. Carvey PM, Hendey B, Monahan AJ. The blood-brain barrier in neurodegenerative disease: a rhetorical perspective. Journal of Neurochemistry 2009;111(2):291-314.

35. Bos PD, Zhang XH, Nadal C, et al. Genes that mediate breast cancer metastasis to the brain. Nature 2009;459(7249):1005-1009.

36. Minn AJ, Gupta GP, Siegel PM, et al. Genes that mediate breast cancer metastasis to lung. Nature 2005;436(7030):518-524.

37. Gupta GP, Nguyen DX, Chiang AC, et al. Mediators of vascular remodelling co-opted for sequential steps in lung metastasis. Nature 2007;446:775-770.

38. Lee BC, Lee TH, Avraham S, Avraham HK. Involvement of the chemokine receptor CXCR4 and its ligand stromal cell-derived factor 1alpha in breast cancer cell migration through human brain microvascular endothelial cells. Molecular Cancer Research: MCR 2004;2:327-338.

39. Lee TH, Avraham HK, Jiang S, Avraham S. Vascular endothelial growth factor modulates the transendothelial migration of MDA- 
MB-231 breast cancer cells through regulation of brain microvascular endothelial cell permeability. The Journal of Biological Chemistry 2003;278:5277-5284.

40. Suzuki HI, Katsura A, Matsuyama H, Miyazono K. MicroRNA regulons in tumor microenvironment. Oncogene 2015;34:3085-3094.

41. Ma L, Young J, Prabhala H, et al. miR-9, a MYC/MYCN-activated microRNA, regulates E-cadherin and cancer metastasis. Nature Cell Biology 2010;12:247-256.

42. Kosaka N, Iguchi H, Hagiwara K, Yoshioka Y, Takeshita F, Ochiya T. Neutral sphingomyelinase 2 (nSMase2)-dependent exosomal transfer of angiogenic microRNAs regulate cancer cell metastasis. The Journal of Biological Chemistry 2013;288:10849-10859.

43. Han M, Wang F, Gu Y, et al. MicroRNA-21 induces breast cancer cell invasion and migration by suppressing smad7 via EGF and TGF- $\beta$ pathways. Oncology Reports 2016;35:73-80.

44. Tavazoie SF, Alarcon C, Oskarsson T, et al. Endogenous human microRNAs that suppress breast cancer metastasis. Nature 2008;451:147-152.

45. Chou J, Lin JH, Brenot A, Kim JW, Provot S, Werb Z. GATA3 suppresses metastasis and modulates the tumour microenvironment by regulating microRNA-29b expression. Nature Cell Biology 2013;15:201213.

46. Zhou W, Fong MY, Min Y, et al. Cancer-secreted miR-105 destroys vascular endothelial barriers to promote metastasis. Cancer Cell 2014;25:501-515.

47. Loriot A, Van Tongelen A, Blanco J, et al. A novel cancer-germline transcript carrying pro-metastatic miR-105 and TET-targeting miR767 induced by DNA hypomethylation in tumors. Epigenetics: Official Journal of the DNA Methylation Society 2014;9:1163-1171.
48. Ma L, Teruya-Feldstein J, Weinberg RA. Tumour invasion and metastasis initiated by microRNA-10b in breast cancer. Nature 2007;449:682-688.

49. Singh R, Pochampally R, Watabe K, Lu Z, Mo YY. Exosome-mediated transfer of miR-10b promotes cell invasion in breast cancer. Molecular Cancer 2014;13:256.

50. Fong MY, Zhou W, Liu L, et al. Breast-cancer-secreted miR-122 reprograms glucose metabolism in premetastatic niche to promote metastasis. Nature Cell Biology 2015;17:183-194.

51. Weil RJ, Palmieri DC, Bronder JL, Stark AM, Steeg PS. Breast cancer metastasis to the central nervous system. The American Journal of Pathology 2005;167:913-920.

52. Bates SE. Central nervous system metastasis from breast cancer. The Oncologist 2015;20:3-4.

53. Chambers AF, Groom AC, MacDonald IC. Dissemination and growth of cancer cells in metastatic sites. Nature Reviews Cancer 2002;2:563572.

54. Kienast $Y$, von Baumgarten $L$, Fuhrmann $M$, et al. Real-time imaging reveals the single steps of brain metastasis formation. Nature Medicine 2010;16:116-122.

55. Heyn C, Ronald JA, Ramadan SS, et al. In vivo MRI of cancer cell fate at the single-cell level in a mouse model of breast cancer metastasis to the brain. Magnetic Resonance in Medicine 2006;56:1001-1010.

56. Valiente $\mathrm{M}$, Obenauf $\mathrm{AC}$, Jin $\mathrm{X}$, et al. Serpins promote cancer cell survival and vascular co-option in brain metastasis. Cell 2014;156:10021016.

57. Yepes M, Sandkvist M, Wong MK, et al. Neuroserpin reduces cerebral infarct volume and protects neurons from ischemia-induced apoptosis. Blood 2000;96:569-576. 